\title{
Time decay of auditory stream biasing
}

\author{
MICHAEL W. BEAUVOIS \\ Université René Descartes, Paris, France \\ and IRCAM, Paris, France \\ and \\ RAY MEDDIS \\ University of Technology, Loughborough, England
}

\begin{abstract}
In an experiment designed to investigate the time decay of auditory stream biasing (ASB), subjects were required to listen to a $10-\mathrm{sec}$ induction sequence of repeated tones (AAAA ...) designed to bias the listener's percept toward hearing an A stream. The induction sequence was followed immediately by a silent interval $(0-8 \mathrm{sec})$, and then a short $\mathrm{ABAB}$... test sequence. To measure the amount of ASB remaining at the end of the silent interval, subjects were asked to indicate whether the test sequence was temporally coherent or had segregated into separate A and B streams. A plot of the mean number of segregation responses against silent-interval duration indicated that the overall time decay of ASB can be described by an exponential decay function with a time constant of $\tau=3.84 \mathrm{sec}$, with musicians having a longer time constant $(\tau=7.84 \mathrm{sec})$ than nonmusicians $(\tau=1.42 \mathrm{sec})$. The length of the time constants for musicians and nonmusicians suggests that the mechanism responsible for ASB is associated with long auditory storage and that future experiments investigating auditory streaming phenomena should use interstimulus intervals of at least $8 \mathrm{sec}$.
\end{abstract}

If an alternating-tone sequence composed of two pure tones of different frequencies ( $\mathrm{A}$ and $\mathrm{B}$ ) is repeated continuously $(\mathrm{ABAB} \ldots$...) , one of two percepts can be heard. At a long tone-repetition time (TRT, or the time interval between the onset of consecutive tones in the sequence), the listener perceives the sequence as a connected series of tones, or a musical trill, with a rhythm determined by the TRT. This property of continuity, where the tone sequence gives a subjective impression of a connected series of tones that is ordered in time, is known as temporal coherence (van Noorden, 1975). However, at short TRTs, or when there is a large frequency separation between the tones $(\Delta f)$, the trill seems to split into two parallel sequences, or "streams," one high and one low in pitch, as if there were two different and unrelated sound sources. Here, the observer's attention will be focused on only one of the tones (A or B), and the stimulus will appear to have a longer periodicity equal to twice the TRT. This phenomenon has become known as auditory stream segregation, where each of the separate sound sources is referred to as a stream (Bregman \& Campbell, 1971).

The perception of auditory streams in alternating-tone sequences is influenced by several factors. Van Noorden (1975) found that the two major factors are $\Delta f$ and TRT. If TRT is small, tones of similar frequencies cluster together to form perceptual streams. In addition to this, if,

This research was supported by a grant to the first author from the Fyssen Foundation, Paris, France. The authors thank Steve McAdams, Valter Ciocca, Nelson Cowan, and, in particular, Marjorie Leek for their comments on an earlier version of this manuscript. Correspondence should be addressed to M. W. Beauvois, IRCAM, 31, rue StMerri, F-75004 Paris, France (e-mail: beauvois@ircam.fr). for a fixed TRT, tones are moved far enough apart in frequency, they will cluster into streams on the basis of frequency proximity (van Noorden, 1975). Van Noorden (1975) also found that between a $\Delta f$ value where a tone sequence is temporally coherent and a $\Delta f$ value where a tone sequence is segregated, there is a range of $\Delta f$ values where the resulting percept is strongly influenced by the observer's attentional set and effort.

It has been shown that the potential for hearing auditory stream segregation increases over the duration of the stimulus. Anstis and Saida (1985) systematically studied the probability of temporal coherence over the length of a 30-sec $\mathrm{ABAB}$ tone sequence. As the percept alternated irregularly between temporal coherence and stream segregation, subjects were required to press a button indicating what percept they heard at any given time over the course of each trial. Their results indicated that all stimuli, irrespective of the stimulus parameters, begin by sounding temporally coherent and that the probability of stream formation increases steadily over time as a function of sequence duration. Similar results were found by Bregman (1978). These findings imply that the percept of a subject is gradually biased toward hearing stream segregation over the duration of the sequence. This gradual biasing toward hearing stream segregation can be thought of as being due to the action of a stream-formation mechanism.

A number of studies have shown some of the characteristics of the stream-formation mechanism. In one experiment, Bregman (1978) presented subjects with ABCB tone "packages" of 4,8 , or 16 tones (where the tone duration could be varied), which was followed by a fixed 4sec silence. This arrangement of package-silence was presented as a repeating loop over the course of each trial. 
The subjects' task was to find the segregation threshold by altering the global tone duration. It was found that they shortened tone duration so as to increase the speed of the packages. That is, they decreased TRT so that the sequence reached the segregation threshold by the end of each package. The 4-sec silence between tone packages was an arbitrary value chosen by Bregman (1978) that served to reset the stream-formation mechanism so that each tone package reached the segregation threshold without the influence of any previous auditory stream biasing (ASB) created by the auditory system. In a further experiment, Bregman (1978) measured the length of the silent interval between tone packages needed to dissipate the ASB created by the stream-formation mechanism. His results indicated that ASB builds up for at least $6 \mathrm{sec}$ and takes at least $6 \mathrm{sec}$ to decay after the sequence stops.

In a study of ASB, Rogers and Bregman (1993) presented subjects with stimuli that consisted of an induction sequence and a test sequence composed of high $(\mathrm{H})$ or low (L) tones. The induction sequence was made from repetitions of one tone $(\mathrm{H})$ that were equally spaced in time, and the test sequence was an HLH-HLH-HLH pattern. The purpose of the induction sequence was to induce stream segregation in a tone pattern (HLH-HLH-HLH) configured so that it would not normally split into two streams when presented alone. Their results showed that more segregated percepts were produced as the number of $\mathrm{H}$ tones in the induction sequence increased. Their results also showed a roughly monotonic increase in the induction of stream segregation (i.e., ASB) as the number of tone onsets in the induction period increased. When one long tone was presented as the induction sequence, there was no real difference from the control condition, which presented white noise during the induction period. These results imply that there is a buildup in ASB over the course of the induction sequence and that ASB is enhanced as the density of tone onsets in the induction sequence increases.

ASB is explained by Bregman (1990) in terms of a primitive auditory-segregation mechanism. Here, the auditory system gradually accumulates evidence that a tone sequence contains different subsets of sounds with distinct properties and that these subsets should be sorted into separate streams. The relative sluggishness in the biasing and decay response $(\approx 6 \mathrm{sec})$ shown by the streamformation mechanism prevents the auditory system from oscillating wildly among different ways of organizing an acoustic input (Bregman, 1990). However, one aspect of ASB not investigated in previous studies is its rate of decay over time. In this paper, the results of an experiment that address this issue are presented.

If a sequence of tones of the same frequency (A) and duration is presented for a long period of time to a subject (the induction sequence), we would expect a stream to be formed centered on Frequency A. If we then present a short $\mathrm{ABAB}$... tone sequence (the test sequence), immediately after the induction sequence, we would expect the test sequence to decompose into two streams: one composed of A tones, and the other composed of B tones. This will occur because the prior existence of the A stream created by the stream-formation mechanism, combined with the continued presence of $A$ in the test sequence, will maintain a continued biasing toward an A-stream percept. This will result in the induction of stream segregation in the test sequence, as found by Rogers and Bregman (1993).

If, however, a period of silence was inserted between the induction and test sequences, we would expect the Astream bias created by the induction sequence (and the action of the stream-formation mechanism) to decay over the duration of the silent interval. This decay of ASB would then weaken the decomposition of the test sequence into separate $A$ and $B$ streams. In addition to this, we would expect longer silent intervals to produce progressively more decay of ASB. After a long enough silence, no decomposition of the test sequence into separate A and B streams would occur, because the streamformation mechanism will have reset itself due to the absence of an inducing sequence, and any ASB would be extinct. Hence, by varying the duration of the silent interval between the induction and test sequences, a function for the time decay of ASB can be found.

In the experiment described below, subjects were required to listen to a 10-sec induction sequence of repeated tones (AAAA ...), which was followed immediately by a silent interval and then a short test sequence that consisted of an $\mathrm{ABAB}$... tone sequence. The subjects were then asked to press a button indicating whether the test sequence was temporally coherent or had segregated into separate A and B streams. From the results, a graph can be plotted of the mean number of segregation responses against silent-interval duration. We would expect the graph to show a monotonic reduction in the number of segregation responses as the duration of the silent interval increased, which either leveled off to an asymptote or reached extinction after a certain silent-interval duration.

\section{METHOD}

\section{Subjects}

There were 18 subjects between 19 and 30 years of age, all of whom were familiar with the concepts of temporal coherence and stream segregation and who had participated in previous experiments on auditory stream segregation.

\section{Stimuli}

The stimulus format was the same in every trial and consisted of an induction sequence, a silent interval, and a test sequence (see Figure 1). The induction and test sequences were the same in every trial, and the tones used in them all had the same duration $(40 \mathrm{msec})$. Each 40-msec tone had a 5-msec rise-and-fall time, implemented using raised cosine ramps in order to prevent onset and offset clicks. Only the silent interval varied between trials, and each silent-interval duration was presented to the subjects once in each experimental block. All stimuli were presented diotically at $65 \mathrm{~dB}$ SPL.

The induction sequence used was $10 \mathrm{sec}$ of a continuously repeating sequence of pure tones (A), which had a frequency of $1000 \mathrm{~Hz}$ and a TRT of $90 \mathrm{msec}$.

The silent interval that followed had 1 of 17 durations, ranging from 0 to $8 \mathrm{sec}$ in half-second increments. These silent-interval du- 


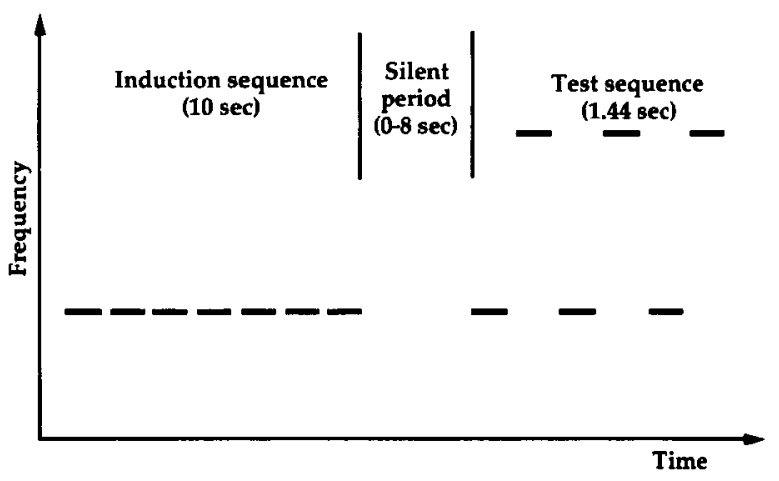

Figure 1. The stimulus configuration used in the experiment.

rations were chosen because Bregman (1978) has shown that the stream-formation mechanism takes at least $6 \mathrm{sec}$ to reset itself and because a pilot study indicated that any decomposition of the test sequence into separate $A$ and $B$ streams was extinct after a silent interval of $8 \mathrm{sec}$ between the induction and test sequences.

The test sequence consisted of eight repetitions of an $\mathrm{AB}$ puretone cycle, where $f_{\mathrm{A}}=1000 \mathrm{~Hz}, f_{\mathrm{B}}=1420 \mathrm{~Hz}$, and TRT $=90 \mathrm{msec}$. The number of $\mathrm{AB}$ repetitions in the test sequence was determined by a preliminary experiment on 3 subjects, who were played a 15 sec version of the above test sequence and were asked to press a button when the $A$ and $B$ tones segregated into separate $A$ and $B$ streams. They completed a total of 150 trials. The average time needed for segregation to occur was $1,437.1 \mathrm{msec}$, approximately equal to eight $\mathrm{AB}$ repetitions $(1,440 \mathrm{msec})$. When presented in isolation, the test sequence always remained coherent. However, if any A-stream bias remained, a segregated percept would result for the test sequence, giving us a measure of any ASB that was present.

\section{Apparatus}

The stimuli were generated on a NeXT computer at a sampling rate of $44.1 \mathrm{kHz}$ using the IRCAM Musical Workstation (Lindemann et al., 1991). The NeXT's digital output was fed into a Digidesign PRO10 D/A converter and then into a Canfort audio amplifier. The audio signal was then relayed to the subjects via Beyer DT-49 headphones while they were seated in a Solona SN1 double-walled sound-attenuation booth.

\section{Procedure}

The subjects were seated in the sound-attenuation booth and shown a copy of Figure 1. They were told that after the induction sequence and silent period, a test sequence would follow for which there would be two possible percepts. One percept was that of a "warble" or "trill," where both tones contributed to the percept (temporal coherence). The other percept was that of "two streams," where either the test sequence had split into separate A and B streams or Tone A stood out more than Tone B (stream segregation). In order to eliminate any effects due to attentional set, the subjects were asked to listen passively to the stimulus and not try to hear either a coherent or a segregated percept. Between the subjects' making their decision and the beginning of the next stimulus, there was a silent interval of $5 \mathrm{sec}$. The subjects were prompted to make their decision and were warned of the next trial by a computer terminal present in the booth. Each subject was given two practice trials before the results were recorded in order to get used to the procedure and the stimuli.

\section{Design}

All 18 subjects completed 10 blocks, each block containing all 17 silent intervals presented in random order. The number of seg- regation responses for each silent-interval value was totaled, and the mean was found. Each mean is therefore based on 180 observations.

\section{RESULTS}

The subject results were pooled, and a graph was plotted of the mean number of segregation responses $(M)$ out of 10 blocks against silent-interval duration $(S)$ in sec (Figure 2). Three functions were fitted to each set of responses: (1) a linear decay, (2) an exponential decay (asymptote $=0$ ), and (3) an exponential decay (asymptote $\neq 0$ ). These gave regression coefficients of $R^{2}=$ $.897, .953$, and .971 , respectively. Hence, the best fit to the data is an exponential decay (asymptote $\neq 0$ ) with the formula

$$
M=2.14+6.1 * \exp (-S / 3.84)
$$

The value of the decay's time constant $(\tau)$ is the divisor of $-S$ (i.e., $\tau=3.84 \mathrm{sec}$ ).

The results were examined further by finding the intersubject correlations between the number of segregation responses for each silent-interval duration. The resultant correlation matrix was then used as the input to a cluster analysis that used the rows of the correlation matrix as the joined variable. The distance was Euclidean, and the complete linkage (farthest neighbor) technique was employed for the analysis. An examination of the results indicated that the subjects' responses fell into three separate groups that differed from each other: G1 (3 subjects), G2 (4 subjects), and G3 (11 subjects). Verbal questioning of the subjects indicated an uneven distribution of subjects who could play a musical instrument across the G1, G2, and G3 groups, with one musician in $\mathrm{G} 1$, none in $\mathrm{G} 2$, and 10 in G3. This suggested plotting two graphs of the mean number of segregation responses

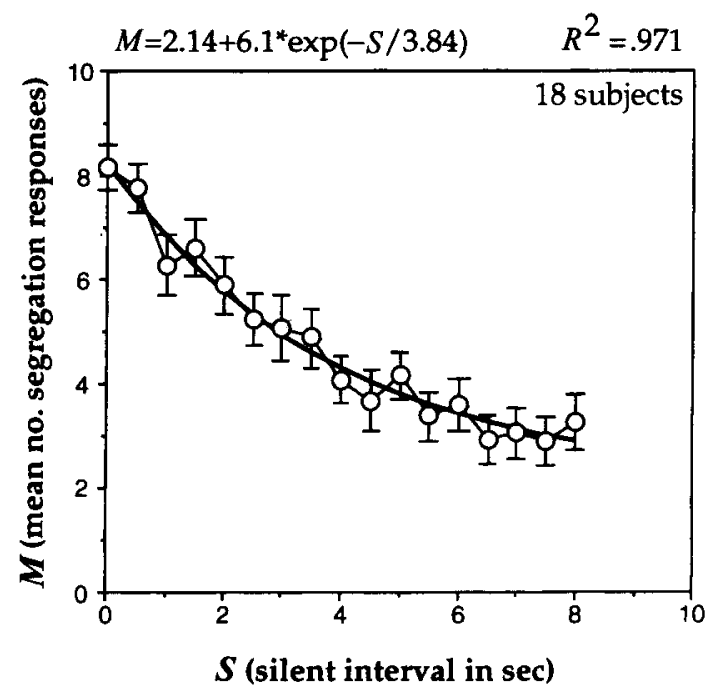

Figure 2. Mean number of segregation responses $(M)$ out of 10 blocks as a function of silent-interval duration $(S)$ for all 18 subjects. Standard error bars are shown for each data point, each data point being based on 180 observations. 

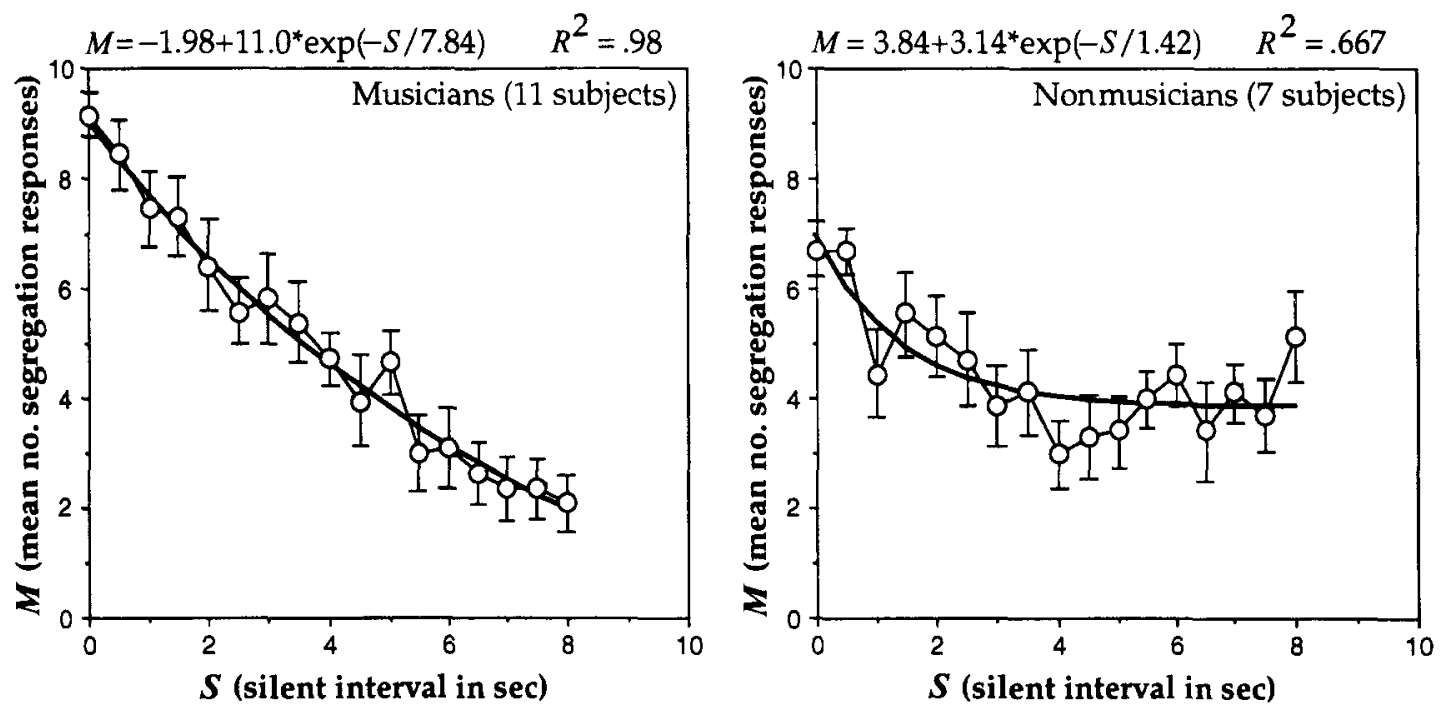

Figure 3. Mean number of segregation responses $(M)$ out of 10 blocks as a function of silent-interval duration $(S)$ for musicians (left) and nonmusicians (right). Standard error bars are shown for each data point. For musicians, each data point is based on 110 observations. For nonmusicians, each data point is based on 70 observations.

against silent-interval duration: one for musicians (Figure 3, left) and one for nonmusicians (Figure 3, right). When the linear and two exponential functions described above were fitted to each set of responses, it was found that the best fit for musicians was an exponential decay (asymptote $\neq 0$ ) with a $\tau$ value of $7.84 \mathrm{sec}$, and the best fit for the nonmusicians was an exponential decay (asymptote $\neq 0$ ) with a $\tau$ value of $1.42 \mathrm{sec}$ (see Figure 3 ).

\section{DISCUSSION}

Figure 3 indicates that both the musicians and the nonmusicians showed an exponential decay of ASB over time, with the time constant of ASB decay for the musicians $(\tau=7.84 \mathrm{sec})$ being greater than that for the nonmusicians $(\tau=1.42 \mathrm{sec})$. However, there are a number of other interesting differences between their responses.

A comparison of the fitted decay curves in Figure 3 indicates that when $S<4 \mathrm{sec}$, the musicians showed more segregation of the test sequence into separate $\mathrm{A}$ and $\mathrm{B}$ streams than did the nonmusicians. This can clearly be seen if we compare the responses of the musicians and the nonmusicians when $S=0 \mathrm{sec}$. Here, we would expect a minimal decay of ASB and, consequently, a high mean number of segregation responses $(M)$. However, when $S=0 \mathrm{sec}$, the nonmusicians showed an ASB peak of $\approx 6.5 \mathrm{M}$, which was less than the musicians' ASB peak of $\approx 9 \mathrm{M}$; this trend of less segregation for the nonmusicians held true up to $S \approx 4 \mathrm{sec}$. However, for $S$ values $>$ $4 \mathrm{sec}$, the nonmusicians showed more segregation of the test sequence than did the musicians.

There could have been a number of reasons for the differences in segregation scores between the musicians and the nonmusicians across $S$. The greater persistence of ASB for the musicians at small $S$ values may have been due to superior auditory-grouping abilities acquired through musical experience. It could also have been due to the musicians' processing the stimuli in an analytical fashion. Due to their practical musical experience, the musicians may have had a stricter definition of a "trill" than did the nonmusicians, and this may have made them more sensitive to any stream segregation that occurred in the test sequence. Evidence to support this suggestion is provided by the results for the nonmusicians. First, the nonmusicians had lower segregation scores than did the musicians when $S<4 \mathrm{sec}$, indicating that the nonmusicians were less susceptible to the effects of ASB than were the musicians. Second, the nonmusicians showed a swift exponential decline of ASB to a near-asymptote level when $S=4 \mathrm{sec}$, indicating that what little ASB the nonmusicians did have reduced quickly. Finally, the segregation scores for the nonmusicians fluctuated in the range of $M=3-5$ when $S<4 \mathrm{sec}$, indicating that, after $S=4 \mathrm{sec}$, the rest of the nonmusicians' responses were approximately evenly divided between "segregated" and "coherent" (i.e., almost at chance level). In contrast, the musicians' segregation scores showed a gradual decline when $S>4 \mathrm{sec}$, indicating a continued sensitivity to ASB decay at long $S$ values. However, although these differences between the results for the musicians and the nonmusicians support the notion that the musicians were more sensitive to any stream segregation that occurred in the test sequence, the differences could simply have been caused by the nonmusicians' not fully understanding the experimental task or not having had enough practice trials beforehand.

The value of the time constant of ASB decay is of great relevance to experimental studies of auditory stream segregation. This is because the ASB created by an experimental stimulus will affect the perception of a fol- 
lowing stimulus if the interstimulus interval is of insufficient duration to reduce any ASB present to a minimal level before the next trial. However, with such marked differences between the responses of musicians and nonmusicians, it is hard to say at what $S$ value the effective cutoff point for ASB decay may lie.

Some idea of the effective cutoff point for ASB decay can be gained by considering the study of Bregman (1978), who observed that the main decay of ASB occurs in the first $4 \mathrm{sec}$ after stimulus offset. Bregman (1978) gave no breakdown of his total subject pool in terms of musicians/nonmusicians. However, if we assume that the proportion of musicians to nonmusicians in his subject pool was similar to ours, then, for a value of $S=4 \mathrm{sec}$, the decay of ASB will be $65 \%$ complete for the total subject pool examined in our study. The nature of exponential decays, which traverse $63 \%$ of their ultimate drop in a time equal to the time constant of the decay, indicates that the exponential ASB decays found in our study will be $63 \%$ complete after a duration of $S=\tau$. The $65 \%$ ASB completion value for our total subject pool not only agrees with Bregman's (1978) observation that the main decay of ASB occurs in the first $4 \mathrm{sec}$ after stimulus offset for the general population but also suggests that the $63 \%$ ASB completion value may well mark the effective cutoff point for ASB decay. That is, the level of residual ASB may be small enough for experimental purposes after a period of silence equal in length to $\tau$. If this is the case, then our results would indicate that ASB decay will be effectively complete at $S=3.84 \mathrm{sec}$ for the total subject pool, $S=7.84 \mathrm{sec}$ for the musicians, and $S=1.42 \mathrm{sec}$ for the nonmusicians. The long time constant of ASB decay for musicians suggests that, to be certain of eliminating any "knock-on" effects of residual ASB, interstimulus intervals in experiments on auditory stream segregation should be at least $8 \mathrm{sec}$ long in order to reduce any ASB created by a stimulus to a minimal level before the next trial.

The phenomenon investigated in this experiment may be related to studies of echoic memory: the retention of the acoustic properties of sounds. Cowan (1984), in an extensive review of the literature, concludes that the evidence of a large number of previous studies indicates that there are two sensory stores with different properties: (1) short auditory storage has a rapidly decaying trace that lasts for 200-300 msec; (2) long auditory storage has a span of 2-10 sec. According to Crowder (1993), the difference between long and short auditory stores is the result of two or more types of information processing by different portions of the auditory nervous system.

The good fit of exponential functions to all the subject data suggests that the time decay of ASB can be described by a leaky integration function. A leaky integrator sums the energy occurring within a given time period or temporal window and functions as a low-pass filter. It shows a gradual exponential accumulation in excitation while a stimulus is on and a gradual exponential decline in excitation once the stimulus has ended. The time constant of the integrator $(\tau)$ controls the accumulation and decline of excitation. If ASB can be described by a leaky integration function, then the overall time constant of ASB decay for the general population (i.e., musicians and nonmusicians) will be $\tau=3.84 \mathrm{sec}$ (see Figure 2). This value for $\tau$ indicates that ASB overlaps with Cowan's (1984) long auditory storage. If this is the case, our experimental results would suggest that the time decay of both ASB and long auditory storage can be modeled using a leaky integration function. Some support for this idea is provided by a study by Lu, Williamson, and Kaufman (1992), who used magnetoencephalographic techniques to measure the response of the primary auditory cortex to tonal stimuli. They found that a subject's memory of the loudness of a sound and the corresponding neuronal response to that sound both decayed exponentially in unison over time. For the 4 subjects they tested, the time constant of the exponential decay was found to vary from 0.8 to $3 \mathrm{sec}$. These time constants are roughly of the same order of magnitude as the overall time constant of the exponential decay of ASB $(\tau=3.84 \mathrm{sec})$ and suggest that the stream-formation mechanism responsible for ASB may be related to auditory memory processes located in the primary auditory cortex.

The convergence of the results of this study with $\mathrm{Lu}$ et al.'s (1992) and Bregman's (1978) findings indicate that the time decay of ASB can be modeled using a leaky integration function. Existing models of auditory stream segregation that use a leaky integrator to reproduce the decay (and accumulation) of ASB over time are those of Beauvois (1992) and Beauvois and Meddis (1991, 1995, 1996). By using the leaky integration principle, these computer models have been able to reproduce a variety of auditory-streaming phenomena, including the results of Anstis and Saida (1985) described above, thereby giving us an insight into the operational characteristics of Bregman's (1990) primitive auditory-segregation mechanism.

\section{REFERENCES}

ANSTIS, S., \& SAIDA, S. (1985). Adaptation to auditory streaming of frequency-modulated tones. Journal of Experimental Psychology: Human Perception \& Performance, 11, 257-271.

BEAUVOIS, M. W. (1992). A computer model of auditory stream segregation. Unpublished doctoral thesis, Loughborough University of Technology, Loughborough, U.K.

BeAuvoIs, M. W., \& Meddis, R. (1991). A computer model of auditory stream segregation. Quarterly Journal of Experimental Psychology, 43A, 517-541.

Beauvois, M. W., \& Meddis, R. (1995). Computer simulation of Gestalt auditory grouping by frequency proximity. In L. S. Smith \& P. J. B. Hancock (Eds.), Neural computation and psychology (pp. 155-164). London: Springer-Verlag.

BEAUVOIS, M. W., \& MEDDIS, R. (1996). Computer simulation of auditory stream segregation in alternating-tone sequences. Journal of the Acoustical Society of America, 99, 2270-2280.

BREGMAN, A. S. (1978). Auditory streaming is cumulative. Journal of Experimental Psychology: Human Perception \& Performance, 4, 380-387.

Bregman, A. S. (1990). Auditory scene analysis. Cambridge, MA: MIT Press. 
BREgman, A. S., \& CAMPBell, J. (1971). Primary auditory stream segregation and perception of order in rapid sequences of tones. Journal of Experimental Psychology, 89, 244-249.

Cowan, N. (1984). On long and short auditory stores. Psychological Bulletin, 96, 341-370.

Crowder, R. G. (1993). Auditory memory. In S. McAdams \& E. Bigand (Eds.), Thinking in sound: The cognitive psychology of human audition (pp. 113-145). Oxford: Oxford University Press.

Lindemann, E., Puckette, M., Viara, E., De Cecco, M., Dechelle, F., \& SMITH, B. (1991). The architecture of the IRCAM Musical Workstation. Computer Music Journal, 15, 41-49.

Lu, Z.-L., Williamson, S. J., \& KaufMan, L. (1992, December 4). Be- havioral lifetime of human auditory sensory memory predicted by physiological measures. Science, 258, 1668-1670.

Rogers, W. L., \& Bregman, A. S. (1993). An experimental evaluation of three theories of auditory stream segregation. Perception \& Psychophysics, 53, 179-189.

VAN NOORDEN, L. P. A. S. (1975). Temporal coherence in the perception of tone sequences. Unpublished doctoral dissertation, Institute for Perception Research, Eindhoven, The Netherlands.

(Manuscript received April 3, 1995; revision accepted for publication March 3, 1996.) 